\title{
Forced Nutation for Rigid Earth Model with Different Theories
}

\author{
Mohamed Soliman ${ }^{1}$, Hadia Hassan Selim ${ }^{1, ~ *, ~ I n a l ~ A d h a m ~ H a s s a n ~}{ }^{2}$ \\ ${ }^{1}$ Department of Astronomy, National Research Institute of Astronomy and Geophysics, Helwan, Egypt \\ ${ }^{2}$ Department of Astronomy and Metrology, Al-Azhar University, Cairo, Egpyt
}

Email address:

hassanselim@yahoo.com (H. H. Selim)

${ }^{*}$ Corresponding author

\section{To cite this article:}

Mohamed Soliman, Hadia Hassan Selim, Inal Adham Hassan. Forced Nutation for Rigid Earth Model with Different Theories. International Journal of Applied Mathematics and Theoretical Physics. Special Issue: Theory and Applications for Rotational Earth and Space Dynamics. Vol. 5, No. 3, 2019, pp. 85-96. doi: 10.11648/j.ijamtp.20190503.16

Received: August 13, 2019; Accepted: September 10, 2019; Published: September 23, 2019

\begin{abstract}
Where Earth is not strictly rigid body but can responds to any effects that tend to its rotation and shape, we will explain, in the present paper, the goal which is to define the forced nutation for a rigid Earth model using two different theories. We will formulate a first order Hamiltonian of a deformable Earth for its rotational motion around the Sun through the contribution of triaxial symmetry of the Earth. The formulation of the theory will be formed twice times. Firstly, deduce the tidal affect's forces by Luni - Solar attraction coupling with the Earth's geopotential force. Secondly, through the formulation, we will neglect the coupling between the different effects (the geopotential Earth force effect and the Luni - Solar attraction force), so, we will find the transform of the Hamiltonian for each force separately. The analytical solution for the formulated Hamiltonian will be derived for the two cases by using perturbation technique of Lie - Hori series. Once can get the analytical solution by getting the generation function, we will derive the nutation series analytically and numerically for each case and conclude over the results.
\end{abstract}

Keywords: Rotation of the Earth, Forced Nutation, Celestial Mechanics

\section{Introduction}

Because of Earth is responds to any effect to be as a deformable body and not as a strictly rigid, it is possesses some sort of elastic properties [1]. Many papers concerned with the study of a rotation for a deformable Earth through different theories of Earth's model as the series of papers [26] and another series [7-13]. So, its shape changes through the rotational motion about its axis, this change affects on the periodic rotation and its uniformity, which intern affects on its shape, also, the same done due to the tidal effects of Sun and Moon.

The present work is concerned with these affects which have been studied twice times, firstly as a coupling effects of rotational Earth including its geopotential and the tidal effects produced with its motion around the Sun through its orbit including the Moon's gravitational effects, secondly, we will concern with neglecting the coupling between the different effects. Short periodic nutation terms will be treated in Hamiltonian form through the contributions of triaxial symmetry of the Earth in each theory and the comparison of results will be done.

\section{Canonical Transformation}

Andoyer and Euler system's variables will be obtained through a canonical transformation [2].

In the case of the body assuming rigid, by taking into account its elasticity such as responds to effects produced by its rotation and by other external tidal effects, we taking into account the Andoyer and Euler angles variables.

As shown in figure 1 , the angles $\lambda, \mu$ and $\nu$ are correspond to Andoyer's variables while $\ell_{m, s}, g_{m, s}$ and $h_{m, s}$ corresponding Delaunay's variables for Moon and Sun respectively.

The conjugate momenta are given by: 


$$
\begin{gathered}
\Lambda=M \cos \varepsilon=P_{\phi}, \\
M=-\frac{P_{\theta}}{\sin \sigma \sin (\psi-v)}, \quad \begin{array}{l}
N=M \cos \sigma=P_{\psi} \\
\Lambda \text { is the component of angular momentum of rotation of }
\end{array} \\
\begin{array}{l}
\text { the deformed body, while } \mathrm{N} \text { is the component on the body } \\
\text { axis OZ [9]. }
\end{array}
\end{gathered}
$$

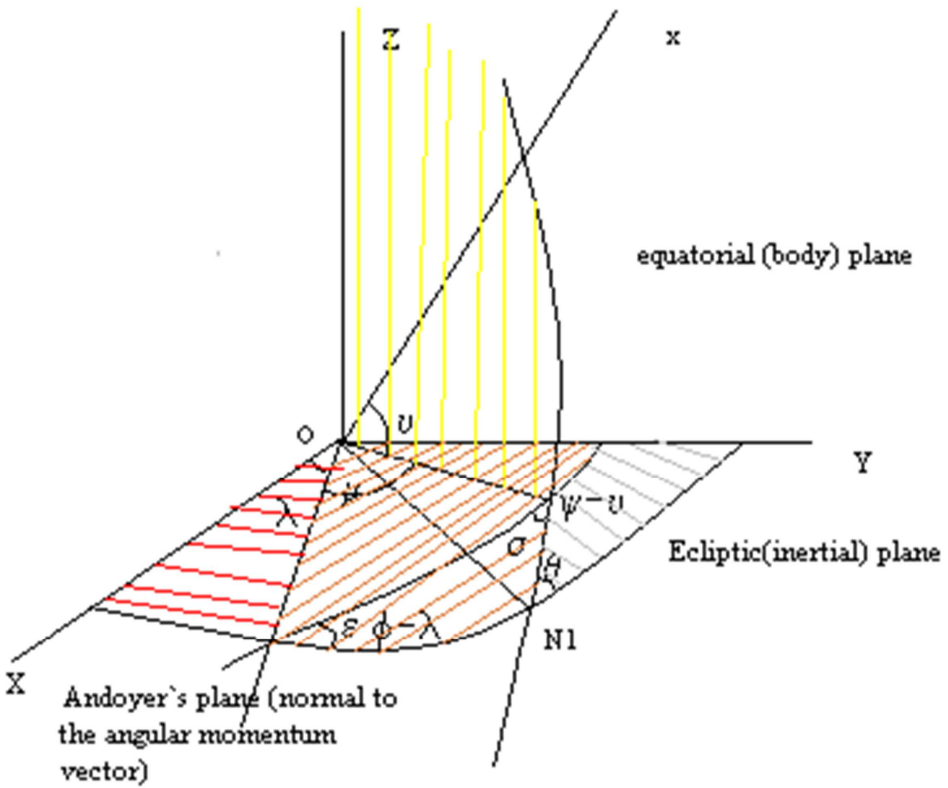

Figure 1. Euler and Andoyer Angles.

\section{The Hamiltonian}

The Hamiltonian $\mathrm{H}$ describing deformable Earth under tidal effects written as [2-4]

$$
H=T_{\circ}+T_{r}+T_{t}+R_{E}+E_{r}+V_{2}+V_{r}
$$

Where

$T_{\circ}$ : is the K. E. for the torque free rigid Earth rotation.

$T_{r}$ : is the increase in the K. E. due to the rotational deformation.

$T_{t}$ : The increase in the $\mathrm{K}$. E due to the tidal deformation.

date.

$E_{r}$ : The elastic strain energy stored by the rotational deformation.

$V_{2}$ : The potential energy due to motion of the disturbing bodies, with assumed triaxial solid.

$V_{r}$ : The increase of potential energy due to the effect of these quantities.

Which defined as:

$$
T_{\circ}=\frac{M^{2}-N^{2}}{2}\left(\frac{\sin ^{2} v}{A}-\frac{\cos ^{2} v}{B}\right)+\frac{N^{2}}{2 c}
$$

$R_{E}$ : The contribution from the motion of the ecliptic of

$$
\begin{gathered}
T_{r}=3 D_{r} \sin \sigma_{r} \frac{M^{2}}{2 c} \sin 2 \sigma\left[\frac{1}{A}+\left(\frac{1}{B}-\frac{1}{A}\right) \cos v_{r} \cos v\right]+\frac{3}{4} \frac{M^{2}-N^{2}}{A B} D_{r} \sin ^{2} \sigma_{r} \sin 2 v_{r} \sin 2 v \\
T_{t}=D_{t}\left\{\left[-\frac{1}{2}(M-N)\left(\frac{1}{A^{2}}+\frac{1}{B^{2}}\right)+\frac{2 N^{2}}{c^{2}}\right] P_{2}\left(\sin \delta^{*}\right)\right. \\
+\frac{1}{4}\left(M^{2}-N^{2}\right)\left(\frac{1}{A^{2}}-\frac{1}{B^{2}}\right)\left(2 P_{2}\left(\sin \delta^{*}\right) \cos 2 v+P_{2}^{2}\left(\sin \delta^{*}\right) \cos 2 \alpha^{*}\right) \\
-\frac{1}{4}\left(M^{2}-N^{2}\right)\left[\left(\frac{1}{A^{2}}+\frac{1}{B^{2}}\right) P_{2}^{2}\left(\sin \delta^{*}\right) \cos 2 \alpha^{*} \cos 2 v-\frac{2}{A B} P_{2}^{2}\left(\sin \delta^{*}\right) \sin 2 \alpha^{*} \sin 2 v\right]
\end{gathered}
$$




$$
\begin{array}{cr}
\left.+\frac{M^{2}}{C} \sin 2 \sigma\left[\frac{1}{A} P_{2}^{1}\left(\sin \delta^{*}\right) \cos \alpha^{*} \sin \nu+\frac{1}{B} P_{2}^{1}(\sin \alpha) \sin \alpha^{*} \cos \nu\right]\right\} & \left(\frac{a^{*}}{r^{*}}\right)^{3} P_{2}(\sin \delta) \cong 3 \sum_{i} B_{i} \cos \theta_{i} \\
R_{E}=M^{\prime} \sin \varepsilon^{\prime} R_{E_{1}}+\Lambda^{\prime} R_{E_{2}} & -3 \sin \sigma \sum_{\tau= \pm 1} \sum_{i} C_{i}(\tau) \cos \left(\mu-\tau \theta_{i}\right)
\end{array}
$$

Where

$$
R_{E_{2}}=(1-\cos \pi) \frac{d \Pi}{d t}
$$

The ecliptic of date is defined by longitude of its node and its inclination $(\Pi, \pi)$ with respect to the ecliptic of epoch, (These two angles being functions of time). The primed variables rotate the ecliptic frame of date to that of the principal axis.

$$
E_{r}=\left(\frac{\Omega}{3}\right)^{2} \frac{2 \pi}{5}\left(I_{2}^{\lambda}+I_{2}^{\mu}\right)
$$

Where: $I_{2}^{\lambda}+I_{2}^{\mu}=5.813114 \times 10^{49}$ g.c.s

$$
\begin{gathered}
V_{2}=\frac{G m^{*}}{r^{* 3}}\left[\frac{2 c-A-B}{2} P_{2}\left(\sin \delta^{*}\right)+\frac{A-B}{4} P_{2}^{2}\left(\sin \delta^{*}\right) \cos 2 \alpha^{*}\right] \\
V_{r}=-\frac{1}{3} k_{2} \frac{\Omega^{2} m^{*} R_{\oplus}^{5}}{r^{* 3}}\left\{P_{2}\left(\sin \delta^{*}\right)+3 \sin \sigma_{r}\left(-\frac{1}{2} \sin \sigma_{r} P_{2}\left(\sin \delta^{*}\right)\right.\right. \\
\quad+\frac{1}{3} \cos \sigma_{r} P_{2}^{1}(\sin \delta) \sin \left(\alpha^{*}+v_{r}\right) \\
\left.\left.-\frac{1}{12} \sin \sigma_{r} P_{2}^{2}\left(\sin \delta^{*}\right) \cos \left(2 \alpha^{*}+2 v_{r}\right)\right)\right\}
\end{gathered}
$$

Where:

$k_{2}$ is the love number, $\alpha^{*}, \delta^{*}$ and $\alpha, \delta$ are spherical coordinates of the perturbing and perturbed body respectively, the spherical functions can be simplified after neglecting the second order terms to

$$
\begin{gathered}
B_{i}=-\frac{1}{6}\left(3 \cos ^{2} I-1\right) \quad A_{i}^{\circ}-\frac{1}{2} \sin 2 I A_{i}^{1)}-\frac{1}{4} \sin ^{2} I A_{i}^{2)} \\
C_{i}(\tau)=-\frac{1}{4} \sin 2 I \quad A_{i}^{\circ}+\frac{1}{2}(1+\tau \cos I)(-1+2 \tau \cos I) A_{i}^{1)}+\frac{\tau}{4} \sin I(1+\tau \cos I) A_{i}^{2)} \\
D_{i}(\tau)=-\frac{1}{2} \sin ^{2} I A_{i}^{\circ}+\tau \sin I(1+\tau \cos I) A_{i}^{1)} \\
-\frac{1}{4}(1+\tau \cos I)^{2} A_{i}^{2)}
\end{gathered}
$$

We taking the orders of magnitude with respect to main

terms $T_{\circ}$ into consideration [6] 


$$
\frac{V_{2}}{T_{\circ}} \cong 6 \times 10^{-8}, \frac{V_{r}}{T_{\circ}} \cong 3 \times 10^{-9}
$$

The perturbation technique used base on the line series method [14].

The Hamiltonian (1) shows that $v, \mu, \ell_{m}, g_{m}, h_{m}$ and $\ell_{s}$ are fast variables, while the remaining variables are slow ones.

In this we shall deal with the fast variables with a first transformation to obtain the generating function for first order terms.

\section{Tidal Effects of Rotational Earth}

Canonical transformation as based as the perturbation's technique Lie series method [14]

$$
\begin{gathered}
H=H\left(u^{\prime}, U^{\prime}\right), \\
H=H_{\circ}+\frac{\varepsilon^{n}}{n !} \sum_{n=1}^{2} H_{n}
\end{gathered}
$$

Where $\mathrm{H}$ written by ordering as

$$
H=H_{\circ}+H_{1}+H_{2}
$$

\subsection{Zero Order}

Since

$$
H_{\circ}=\frac{M^{2}}{4}\left(\frac{1}{A}+\frac{1}{B}\right)+\frac{N^{2}}{4}\left(\frac{2}{c}-\frac{1}{A}-\frac{1}{B}\right)+n_{\ell_{s}} P_{\ell_{s}}+n_{\ell_{m}} P_{\ell_{m}}+n_{g_{m}} P_{g_{m}}+n_{h_{m}} P_{h_{m}}
$$

Transformation of zero order:

$$
H_{\circ}=H_{\circ}^{*}
$$

For simplicity, we will dropping the primes so,

$$
H_{\circ}^{*}=\frac{M^{2}}{4}\left(\frac{1}{A}+\frac{1}{B}\right)+\frac{N^{2}}{4}\left(\frac{2}{c}-\frac{1}{A}-\frac{1}{B}\right)+n_{\ell_{s}} P_{\ell_{s}}+n_{\ell_{m}} P_{\ell_{m}}+n_{g_{m}} P_{g_{m}}+n_{h_{m}} P_{h_{m}}
$$

\subsection{First Order}

$$
\begin{aligned}
H_{1}=\frac{1}{\varepsilon}\left\{R_{E}\right. & +E_{r}+3 D_{r} \frac{M^{2} \sin 2 \sigma}{2 A c} \sin \sigma_{r} \cos \sigma_{r}+\frac{M^{2}-N^{2}}{4}\left(\frac{1}{A}-\frac{1}{B}\right) \cos 2 v \\
& +U_{\circ}\left[\frac{1}{2} \chi_{1}\left(3 \cos ^{2} \sigma-1\right)+3 \chi_{2} \sin ^{2} \sigma+\frac{3}{2} \chi_{3} \sin ^{2} 2 \sigma\right] \\
+ & U_{1}\left[-\frac{1}{2} \chi_{1} \sin 2 \sigma+\chi_{2} \sin 2 \sigma-\chi_{3} \sin 2 \sigma\left(1-\cos ^{2} \sigma\right)\right] \\
& \left.+U_{2}\left[\frac{1}{4} \sin ^{2} \sigma+\frac{1}{2} \chi_{2}\left(1+\cos ^{2} \sigma\right)-\frac{1}{4} \chi_{3} \sin ^{2} 2 \sigma\right]\right\}
\end{aligned}
$$

Where

$$
\begin{aligned}
D_{r} & =-\frac{\Omega^{2}}{3} \frac{4 \pi}{15} \int_{r}\left[2 \rho_{\circ} r^{4}\left(5 F_{2}(r)+r^{2} G_{2}(r)\right)\right. \\
& \left.-r^{5} \frac{d \rho_{\circ}}{d r}\left(2 F_{2}(r)+r^{2} G_{2}(r)\right)\right] d r
\end{aligned}
$$

Which for the Earth model used and taking $\Omega \cong \omega_{3}, D_{r}$ has a value of $D_{r}=-2.845379 \times 10^{41}$ g.c.s 


$$
D_{t}=\frac{G m^{*}}{r^{* 3}} \frac{2 \pi}{15} \int_{r}\left[2 \rho_{\circ} r^{4}\left(5 F_{2}(r)+r^{2} G_{2}(r)\right)-r^{5} \frac{d \rho_{\circ}}{d r}\left(2 F_{2}(r)+r^{2} G_{2}(r)\right)\right] d r
$$

The function $\rho_{\mathrm{o}}, F_{2}$ and $G_{2}$ depend on the model used.

$$
D_{t}= \begin{cases}6.95339 \times 10^{36} \text { g.c.s } & \text { for Moon } \\ 3.185508 \times 10^{36} \text { g.c.s } & \text { for Sun }\end{cases}
$$

we rewrite eqns. (5) in a simplest form as

$$
H_{1}=\sum_{n=0}^{1} \sum_{p=0}^{2} \sum_{\tau=-1}^{1} \sum_{i} \xi_{n p \tau i} \cos \left(2 n v+p \mu+\tau \theta_{i}\right)
$$

Where

$$
\begin{gathered}
\xi_{0000}=\frac{1}{\varepsilon}\left\{\mathrm{R}_{\mathrm{E}}+\mathrm{E}_{\mathrm{r}}+3 \mathrm{D}_{\mathrm{r}}\left(\mathrm{M}^{2} \sin 2 \sigma / 2 \mathrm{Ac}\right) \sin \sigma_{\mathrm{r}} \cos \sigma_{\mathrm{r}}\right\} \\
\xi_{1000}=\frac{1}{\varepsilon}\left\{\frac{M^{2}-N^{2}}{4}\left(\frac{1}{A}-\frac{1}{B}\right) \cos 2 v\right\} \\
\xi_{01 i 0}=\frac{B_{i}}{\varepsilon}\left\{\frac{1}{2} \chi_{1}\left(3 \cos ^{2} \sigma-1\right)+3 \chi_{2} \sin ^{2} \sigma+\frac{3}{2} \chi_{3} \sin ^{2} 2 \sigma\right\} \\
\xi_{00,-1 i}=0 \\
\xi_{01,-1 i}=\frac{c_{i}(-1)}{\varepsilon}\left\{-\frac{1}{2} \chi_{1} \sin 2 \sigma+\chi_{2} \sin 2 \sigma-\chi_{3} \sin 2 \sigma\left(1-2 \cos ^{2} 2 \sigma\right)=\frac{C_{i}(1)}{\left.C_{i}(-1)\right)} \xi_{011 i}\right\} \\
\xi_{02,-1 i}=\frac{D_{i}(-1)}{\varepsilon}\left\{\frac{1}{4} \chi_{1} \sin ^{2} \sigma+\frac{1}{2} \chi_{2}\left(1+\cos ^{2} \sigma\right)-\frac{1}{4} \chi_{3} \sin ^{2} 2 \sigma\right\}=\frac{D_{i}(1)}{D_{i}(-1)} \xi_{021 i} \\
C_{i}(0)=D_{i}(0)=0 \quad H_{1 p}=\tilde{H}_{1}-H_{1}^{*}
\end{gathered}
$$

Transformation of first order:

We have

Then from eqns. (36) and (37)

$$
H^{*}=\tilde{H}_{1}+\left(H_{\circ}, W_{1}\right)
$$

From (27)

$$
\tilde{H}_{1}=\sum_{n=0}^{1} \sum_{p=0}^{2} \sum_{\tau=-1}^{1} \sum_{i} \xi_{n p \tau i} \cos \left(2 n v+p \mu+\tau \theta_{i}\right)
$$

We choose

$$
H_{1 p}=\sum_{n=0}^{1} \sum_{p=0}^{2} \sum_{\tau=-1}^{1} \sum_{i \neq 0} \xi_{n p \tau i}^{\prime} \cos \left(2 n v+p \mu+\tau \theta_{i}\right)
$$

$$
\begin{gathered}
\xi_{0000}^{\prime}=0 \\
\xi_{001 i}^{\prime}=\xi_{100 i} \\
\zeta_{01,-1 i}^{\prime}=\zeta_{01,-1 i} \\
\zeta_{02,-1 i}^{\prime}=\zeta_{02,-1 i}
\end{gathered}
$$$$
H_{1}^{*}=\left\langle\tilde{H}_{1}\right\rangle_{\mu, v, \ell_{1}, g_{1}, h_{1}, \ell_{2}}
$$

Then

Since

$$
H_{1}^{*}=\xi_{0000}+\xi_{0010}
$$

$$
H_{1 p}=\left(W_{1}, H_{\circ}\right)
$$

Since 


$$
=\frac{\partial W_{1}}{\partial \mu} \frac{\partial H_{\circ}}{\partial M}+\frac{\partial W_{1}}{\partial v} \frac{\partial H_{\circ}}{\partial N}+\frac{\partial W_{1}}{\partial \ell_{m}} \frac{\partial H_{\circ}}{\partial P_{\ell_{m}}}+\frac{\partial W_{1}}{\partial g_{m}} \frac{\partial H_{\circ}}{\partial p_{g_{m}}}+\frac{\partial W_{1}}{\partial h_{1}} \frac{\partial H_{\circ}}{\partial p_{h_{m}}}+\frac{\partial W_{1}}{\partial \ell_{s}} \frac{\partial H_{\circ}}{\partial p_{\ell_{2}}}
$$

from eqns. (38) and (43)

$$
W_{1}=\sum_{n=0}^{1} \sum_{p=0}^{2} \sum_{\tau=-1}^{1} \sum_{i=0} \frac{\xi_{n p \tau i}^{\prime}}{Q} \sin \left(2 n v+p \mu+\tau \theta_{i}\right)
$$

Where

$$
\begin{gathered}
Q=2 n_{v}+p_{\mu}+\tau n_{i}, \\
n_{\mu}=\frac{\partial H_{\circ}}{\partial N}=\frac{N}{2}\left(\frac{2}{c}-\frac{1}{A}-\frac{1}{B}\right), \\
n_{v}=\frac{\partial H_{\circ}}{\partial M}=\frac{M}{2}\left(\frac{1}{A}+\frac{1}{B}\right), \\
n_{i}=\frac{\partial \theta_{i}}{\partial t}
\end{gathered}
$$

Since the angle $\sigma$ between the angular momentum vector and the figure axis of Earth is about $10^{-6} \mathrm{rad}$, we could take the approximation $\cos \sigma \cong 1$ [5].

\subsection{Forced Nutation}

Once the generating function has been developed, the periodic perturbation of the fundamental plane, which includes the Andoyer plane (the plane perpendicular to the angular momentum vector) and the equatorial plane (the

plane perpendicular to figure axis of Earth) can be obtained.

\subsubsection{Nutation of the Andoyer Plane}

The longitude of the node and the inclination of this plane are given respectively by $\lambda$ and $\mathrm{I}, I=\cos ^{-1}\left(\frac{\Lambda}{M}\right)$. The nutation corresponding to the variables, known as poisson terms, are obtained through the equation [15-16].

$$
\begin{gathered}
\Delta \lambda=-\frac{1}{M \sin I} \frac{\partial W}{\partial I} \\
\Delta I=\frac{1}{M \sin I}\left(\frac{\partial W}{\partial \lambda}-\cos I \frac{\partial W}{\partial \mu}\right)
\end{gathered}
$$

From eqns. (44) and (46) we have

$$
\Delta \lambda=\frac{1}{M \sin I} \sum_{n=0}^{1} \sum_{p=o}^{2} \sum_{\tau=-1}^{1} \sum_{i \neq 0} \frac{\xi_{n p \tau i, I}^{\prime}}{Q} \cos \left(2 n v+p \mu+\tau \theta_{i}\right)
$$

$$
\Delta I=\frac{1}{M \sin I} \sum_{n=0}^{1} \sum_{p=0}^{2} \sum_{\tau=-1}^{1} \sum_{i \neq 0} \frac{\xi_{n p \tau i}^{\prime}}{Q}\left(m_{5}-p \cos I\right) \cos \left(2 n v+p \mu+\tau \theta_{i}\right)
$$

\subsubsection{Nutation of Equatorial Plane}

The longitude of the node, $\psi_{f}=-\lambda$ and the inclination $\varepsilon_{f}=-I_{f}$ (in-phase) are given up to the first order by [15]

$\lambda_{f}=\lambda+\frac{\sigma}{\sin I} \sin \mu$ and $I_{f}=I+\sigma \cos \mu$ following Kinoshita, the periodic perturbation of the increment $\lambda_{f}-\lambda$ and $I_{f}-I$ called oppolzer terms, are given up to the first order by

$$
\begin{aligned}
& \Delta\left(\lambda_{f}-\lambda\right)=\frac{1}{M \sin \sigma \sin I}\left[\left(\sin \mu \frac{\partial W}{\partial v}-\frac{\partial W}{\partial \mu}\right)+\sigma \cos \mu \frac{\partial W}{\partial \sigma}\right], \\
& \Delta\left(I_{f}-I\right)=\frac{1}{M \sin \sigma \sin I}\left[\left(\cos \mu \frac{\partial W}{\partial v}-\frac{\partial W}{\partial \mu}\right)-\sigma \sin \mu \frac{\partial W}{\partial \sigma}\right]
\end{aligned}
$$

From eqns. (44) and (49)

$$
\Delta\left(\lambda_{f}-\lambda\right)=\frac{1}{M \sin \sigma \sin I}\left[\sum _ { n = 0 } ^ { 1 } \sum _ { p = 0 } ^ { 2 } \sum _ { \tau = - 1 } ^ { 1 } \sum _ { i \neq 0 } \left\{\sin \mu(2 n-p) \frac{\xi_{n p \tau i}^{\prime}}{Q} \cos \left(2 n v+p \mu+\tau \theta_{i}\right)\right.\right.
$$




$$
\begin{gathered}
\left.\left.+\sigma \cos \mu \frac{\xi_{n p \tau i, \sigma}^{\prime}}{Q} \sin \left(2 n v+p \mu+\tau \theta_{i}\right)\right\}\right] \\
\Delta\left(I_{f}-I\right)=\frac{1}{M \sin \sigma \sin I}\left[\sum _ { n = 0 } ^ { 1 } \sum _ { p = 0 } ^ { 2 } \sum _ { \tau = - 1 } ^ { 1 } \sum _ { i \neq 0 } \left\{\cos \mu(2 n-p) \frac{\xi_{n p \tau i}^{\prime}}{Q} \cos \left(2 n v+p \mu+\tau \theta_{i}\right)\right.\right. \\
\left.\left.+\sigma \sin \mu \frac{\xi_{n p \tau i, \sigma}^{\prime}}{Q} \sin \left(2 n v+p \mu+\tau \theta_{i}\right)\right\}\right]
\end{gathered}
$$

\subsection{Final Expressions}

Bu using eqns. (47), (48), (50) and (51) we can find

$$
\begin{gathered}
\Delta \psi_{f}=-\frac{1}{M Q \sin I} \sum_{n=0}^{1} \sum_{p=0}^{2} \sum_{\tau=-1}^{1} \sum_{i \neq 0}\left\{\sin \mu(p-2 n) \frac{\xi_{n p \tau i}^{\prime}}{\sin \sigma} \cos \left(2 n v+p \mu+\tau \theta_{i}\right)\right. \\
\left.-\sigma \cos \mu \frac{\xi_{n p \tau i, \sigma}^{\prime}}{\sin \sigma} \sin \left(2 v+p \mu+\tau \theta_{i}\right)+\xi_{n p \tau i, I}^{\prime} \sin (n v+2 p v+\tau \theta)\right\} \\
\Delta I_{f}=-\frac{1}{M Q} \sum_{n=0}^{1} \sum_{p=0}^{2} \sum_{\tau=-1}^{1} \sum_{i \neq 0}\left\{\cos \mu(2 n-p) \frac{\xi_{n p \tau i}^{\prime}}{\sin \sigma} \cos \left(2 n v+p \mu+\tau \theta_{i}\right)\right. \\
\left.+\sigma \cos \mu \frac{\xi_{n p \tau i, \sigma}^{\prime}}{\sin \sigma} \sin \left(2 n v+p \mu+\tau \theta_{i}\right)+\left(m_{5}-p \cos I\right) \frac{\xi_{n p \tau i, I}^{\prime}}{\sin I} \cos (2 n v+2 p v+\tau \theta)\right\}
\end{gathered}
$$

\section{Forced Nutation and the Free Earth Rotation}

In the following, we shall focus on two affects on the rigid Earth, the centrifugal perturbation and the tidal perturbation forces by neglect the coupling between them.

\subsection{Free Rigid Earth}

Let the Hamiltonian forced as

$$
H_{f r}=H_{\circ f r}+H_{1 f r}+H_{2 f r}
$$

Where

$$
\begin{gathered}
H_{\circ \circ r}=T_{\circ}^{0} \\
H_{1 f r}=T_{\circ}^{1}+V_{2}^{1}+R_{E} \\
H_{2 f r}=V_{2}^{2}
\end{gathered}
$$

Where;

$$
\begin{gathered}
T_{\circ}=T_{\circ}^{0}+T_{\circ}^{1}, \\
T_{\circ}^{0}=\frac{M^{2}}{4}\left(\frac{1}{A}+\frac{1}{B}\right)+\frac{N^{2}}{4}\left(\frac{2}{c}-\frac{1}{A}-\frac{1}{B}\right),
\end{gathered}
$$

$$
\begin{aligned}
& T_{\circ}^{1}=\frac{M^{2}-N^{2}}{4}\left(\frac{1}{B}-\frac{1}{A}\right) \cos 2 v . \\
& V_{2}^{1}=\frac{G m^{*}}{r^{* 3}} \frac{2 c=A=B}{2} P_{2}\left(\sin \delta^{*}\right)
\end{aligned}
$$

Zero order transformation:

$$
\begin{aligned}
& H_{\circ f r}=\frac{M^{2}}{4}\left(\frac{1}{A}+\frac{1}{B}\right)+\frac{N^{2}}{4}\left(\frac{2}{c}-\frac{1}{A}-\frac{1}{B}\right) \\
& H_{\circ f r}^{*}=\frac{M^{2}}{4}\left(\frac{1}{A}+\frac{1}{B}\right)+\frac{N^{2}}{4}\left(\frac{2}{c}-\frac{1}{A}-\frac{1}{B}\right)
\end{aligned}
$$

The primes are dropped for simplicity of writing. First order transformation:

$$
\begin{gathered}
H_{1 f r}=R_{E}+\frac{M^{2}-N^{2}}{4}\left(\frac{1}{B}-\frac{1}{A}\right) \cos 2 v \\
+\frac{1}{2} k_{\circ}^{\prime}\left(3 \cos ^{2} \sigma-1\right) \sum_{i} B_{i} \cos \theta_{i}
\end{gathered}
$$

Where

$$
k_{\circ}^{\prime}=\frac{3 G m^{*}}{a^{* 3}} \frac{2 c-A-B}{2}
$$


$\mathrm{G}$ is the gravitational constant, $m^{*}, a^{*}$ are the mass and the semi-major axis of the perturbing body (Moon, Sun) and A, $\mathrm{B}, \mathrm{c}$ are the moments of inertia in the absence of deformation (we will use triaxial symmetry case $\mathrm{A}{ }_{\mathrm{B}}$ ).

So

$$
\begin{gathered}
k_{\circ}^{\prime}=\frac{3 G m^{*}}{r^{* 3}}(c-A) \\
H_{1 f r}^{*}=R_{E}+\frac{1}{2} k_{\circ}^{\prime}\left(3 \cos ^{2} \sigma-1\right) B_{\circ}^{*} \\
H_{1 f r, \text { periodic }}=\frac{M^{2}-N^{2}}{4}\left(\frac{1}{B}-\frac{1}{A}\right) \cos 2 v \\
w_{1 f r}=\frac{M^{2}-N^{2}}{4}\left(\frac{1}{B}-\frac{1}{A}\right) \frac{\sin 2 v}{2 n_{v}}+\frac{1}{2} k_{\circ}^{\prime}\left(3 \cos ^{2} \sigma-1\right) \sum_{i \neq 0} \frac{B_{i}}{n_{i}} \sin \theta_{i}
\end{gathered}
$$

The generating function of this transformation will be

\subsection{Tidal Perturbation Force}

We will study the effects of tidal forces, rotational and deformation so, let the Hamiltonian formed as

$$
\begin{gathered}
H_{t}=H_{\circ t}+H_{1 t}+H_{2 t} \\
H_{\circ}=0 \\
H_{1 t}=E_{r}+T_{r}^{1}+T_{t}^{1}+V_{r}^{1} \\
H_{1 t}=E_{r}+3 D_{r} \frac{M^{2} \sin 2 \sigma}{2 A c} \sin \sigma_{r} \cos \sigma_{r} \\
+U_{\circ}\left[\frac{1}{2} \chi_{1}\left(3 \cos ^{2} \sigma-1\right)+3 \chi_{2} \sin ^{2} \sigma+\frac{3}{2} \chi_{3} \sin ^{2} 2 \sigma\right] \\
+U_{1}\left[-\frac{1}{2} \chi_{1} \sin 2 \sigma+\chi_{2} \sin 2 \sigma-\chi_{3} \sin 2 \sigma\left(1-2 \cos ^{2} \sigma\right)\right] \\
+U_{2}\left[\frac{1}{4} \chi_{1} \sin ^{2} \sigma+\frac{1}{2}\left(1+\cos ^{2} \sigma\right)-\frac{1}{4} \chi_{3} \sin ^{2} 2 \sigma\right]
\end{gathered}
$$

We do not need to $H_{2 t}$, since we calculate the first order transformation

First order transformation:

$$
\begin{gathered}
H_{1 t}^{*}=E_{r}+\frac{3 D_{r} M^{2} \sin 2 \sigma}{2 A c} \sin \sigma_{r} \cos \sigma_{r} \\
+B_{\circ}\left[\frac{1}{2} \chi_{1}\left(3 \cos ^{2} \sigma-1\right)+3 \chi_{2} \sin ^{2} \sigma+\frac{3}{2} \chi_{3} \sin ^{2} 2 \sigma\right]
\end{gathered}
$$

and the periodic terms are:

$$
H_{1 t, p e r}=B_{i \neq 0}\left[\frac{1}{2} \chi_{1}\left(3 \cos ^{2} \sigma-1\right)-\frac{1}{2} k_{\circ}^{\prime}\left(3 \cos ^{2} \sigma-1\right)+3 \chi_{2} \sin ^{2} \sigma+\frac{3}{2} \chi_{3} \sin ^{2} 2 \sigma\right] \cos \theta_{i}
$$




$$
\begin{array}{r}
+C_{i}(\tau)\left[-\frac{1}{2} \chi_{1} \sin 2 \sigma+\chi_{2} \sin 2 \sigma-\chi_{3} \sin 2 \sigma\left(1-2 \cos ^{2} \sigma\right)\right] \cos \left(\mu-\tau \theta_{i}\right) \\
+D_{i}(\tau)\left[\frac{1}{4} \chi_{1} \sin ^{2} \sigma+\frac{1}{2} \chi_{2}\left(1+\cos ^{2} \sigma\right)-\frac{1}{4} \chi_{3} \sin ^{2} 2 \sigma\right] \cos \left(2 \mu-\tau \theta_{i}\right)
\end{array}
$$

where

$$
H_{\text {periocic }}=\tilde{H}-H^{*}
$$

And

$$
H_{\text {periodic }}=\left(W_{1}, H_{\circ}\right)
$$

after transformation eqn. (74), we can get the generating function as follows for simplification

$$
\begin{aligned}
& W_{1 t}=\int H_{1 t, \text { periodic }} d t \\
& =W_{10 t}+W_{11 t}+W_{12 t}
\end{aligned}
$$

Where

$$
\begin{gathered}
W_{10 t}=\left[\frac{1}{2} \chi_{1}\left(3 \cos ^{2} \sigma-1\right)-\frac{1}{2}\left(3 \cos ^{2}-1\right) k_{\circ}^{\prime}+3 \chi_{2} \sin ^{2} \sigma+\frac{3}{2} \chi_{3} \sin ^{2} 2 \sigma\right] \sum_{i \neq 0} \frac{B_{i}}{n_{i}} \sin \theta_{i} \\
W_{11 t}=\left[-\frac{1}{2} \chi_{1} \sin 2 \sigma+\chi_{2} \sin 2 \sigma-\chi_{3}\left(1-2 \cos ^{2} \sigma\right) \sin 2 \sigma\right] \sum_{\tau=-1}^{1} \sum_{i \neq 0} \frac{C_{i}(\tau)}{n_{\mu}-\tau n_{i}} \sin \left(\mu-\tau \theta_{i}\right) \\
W_{12 t}=\left[\frac{1}{4} \chi_{1} \sin ^{2} \sigma+\frac{1}{2} \chi_{2}\left(1+\cos ^{2} \sigma\right)-\frac{1}{4} \chi_{3} \sin ^{2} 2 \sigma\right] \sum_{\tau=-1}^{1} \sum_{i \neq 0} \frac{D_{i}(\tau)}{2 n_{\mu}-\tau n_{i}} \sin \left(2 \mu-\tau \theta_{i}\right)
\end{gathered}
$$

\subsection{Forced Nutation}

\subsubsection{Nutation of Andoyer Plane}

By using eqns. (68) and (75) in eqn. (46) respectively, where we can take the approximation by neglecting terms in $\sigma^{2}$, can get

$$
\begin{gathered}
\Delta \lambda_{f r}=-\frac{1}{M \sin I} k_{\circ}^{\prime} \sum_{i \neq 0} \frac{B_{i, I}}{n_{i}} \sin \theta_{i}, \\
\Delta \lambda_{t}=-\frac{1}{M \sin I}\left\{\left(\chi_{1}-k_{\circ}^{\prime}\right) \sum_{i \neq 0} \frac{B_{i, I}}{n_{i}} \sin \theta_{i}+\chi_{2} \sum_{i= \pm 1} \sum_{i \neq 0} \frac{D_{i}(\tau)}{2 n_{\mu}-\tau n_{i}} \sin \left(2 \mu-\tau \theta_{i}\right)\right\} \\
\Delta I_{f r}=-\frac{k_{\circ}^{\prime}}{M \sin I} \sum_{i \neq 0} \frac{B_{i}}{n_{i}} m_{5} \cos \theta_{i}, \\
\Delta I_{t}=\frac{1}{M \sin I}\left\{\left(k_{\circ}^{\prime}-\chi_{1}\right) \sum_{i \neq 0} \frac{m_{5} B_{i}}{n_{i}} \cos \theta_{i}-\left(m_{5}-2 \cos I\right) \chi_{2} \sum_{\tau= \pm 1} \sum_{i \neq 0} \sin \left(2 \mu-\tau \theta_{i}\right)\right\}
\end{gathered}
$$

Where

$$
B_{i, I}=\frac{\partial B_{i}}{\partial I}, D_{i, I}=\frac{\partial D_{i}(\tau)}{\partial I}
$$




\subsubsection{Nutation of Equatorial Plane}

By using eqns. (49) throughout (68) and (75) respectively we can get

$$
\begin{gathered}
\Delta\left(\lambda_{f}-\lambda\right)_{f r} \cong 0 \\
\Delta\left(I_{f}-I\right)_{f r} \cong 0 \\
\Delta\left(\lambda_{f}-\lambda\right)_{t}=\frac{1}{M \sin I}\left[\frac{1}{2}\left(\chi_{2}-\frac{1}{2} \chi_{1}\right) \sum_{\tau= \pm 1} \sum_{i \neq 0} \frac{C_{i}(\tau)}{n_{\mu}-\tau n_{i}} \sin \left(2 \mu-\tau \theta_{i}\right)\right. \\
+\frac{3}{2}\left(\frac{1}{2} \chi_{1}-\chi_{2}\right) \sum_{\tau= \pm 1} \sum_{i \neq 0} \frac{C_{i}(\tau)}{n_{\mu}-\tau n_{i}} \sin \left(2 \mu-\tau \theta_{i}\right) \\
\left.-\frac{1}{4}\left(\chi_{1}+4 \chi_{2}-\chi_{3}\right) \sum_{\tau- \pm 1} \sum_{i} \frac{D_{i}(\tau)}{n_{\mu}-\tau n_{i}}\left(\sin \left(3 \mu-\tau \theta_{i}\right)+\sin \left(\mu-\tau \theta_{i}\right)\right)\right] \\
\Delta\left(I_{f}-I\right)_{t}=\frac{1}{M}\left[\frac{1}{2}\left(\chi_{2}-\frac{1}{2} \chi_{1}\right) \sum_{\tau= \pm 1} \sum_{i \neq 0} \frac{C_{i}(\tau)}{n_{\mu}-\tau n_{i}} \cos \left(2 \mu-\tau \theta_{i}\right)\right. \\
+\frac{3}{2}\left(\frac{1}{2} \chi_{1}-\chi_{2}\right) \sum_{\tau= \pm 1} \sum_{i \neq 0} \frac{C_{i}(\tau)}{n_{\mu}-\tau n_{i}} \cos \tau \theta_{i} \\
\left.-\frac{1}{4}\left(\chi_{1}+4 \chi_{2}-\chi_{3}\right) \sum_{\tau= \pm 1} \sum_{\neq 0} \frac{D_{i}(\tau)}{2 n_{\mu}-\tau n_{i}}\left(\cos \left(3 \mu-\tau \theta_{i}\right)+\cos \left(\mu-\tau \theta_{i}\right)\right)\right]
\end{gathered}
$$

\subsubsection{Final Expression}

By using eqns. (79), (80), (82) and (83), we can calculate the nutation series in longitude $\Delta \psi_{f}$ and obliquity $\Delta I_{f}$ of the figure plane:

$$
\begin{gathered}
\Delta \psi_{f_{f r}}=-\frac{1}{M \sin I} k_{\circ}^{\prime} \sum_{i \neq 0} \frac{B_{i, I}}{n_{i}} \sin \theta_{i} \\
\Delta \psi_{f_{t}}=-\frac{1}{M \sin I}\left\{\left[\left(\chi_{1}-k_{\circ}^{\prime}\right) \sum_{\tau= \pm} \sum_{i \neq 0} \frac{B_{i, I}}{n_{i}}-\frac{3}{2}\left(\frac{1}{2} \chi_{1}-\chi_{2}\right) \sum_{\tau= \pm 1} \sum_{i \neq 0} \frac{C_{i}(\tau)}{n_{\mu}-\tau n_{i}}\right] \sin \tau \theta_{i}\right. \\
+\left[\chi_{2} \sum_{\tau= \pm 1} \sum_{i \neq 0} \frac{D_{i, I}(\tau)}{2 n_{\mu}-\tau n_{i}}-\frac{1}{2}\left(\chi_{2}-\frac{1}{2} \chi_{1}\right) \sum_{\tau= \pm 1} \sum_{i \neq 0} \frac{C_{i}(\tau)}{n_{\mu}-\tau n_{i}}\right] \sin \left(2 \mu-\tau \theta_{i}\right) \\
\left.+\frac{1}{4}\left(\chi_{1}+4 \chi_{2}-\chi_{3}\right) \sum_{\tau= \pm 1} \sum_{i \neq 0} \frac{D_{i}(\tau)}{2 n_{\mu}-\tau n_{i}}\left(\sin \left(3 \mu-\tau \theta_{i}\right)+\sin \left(\mu-\tau \theta_{i}\right)\right)\right\} \\
\Delta I_{f_{t}=} \frac{1}{M}\left\{\left[\left(k_{\circ}^{\prime}-\chi_{1}\right) \sum_{\tau= \pm 1} \sum_{i \neq 0} \frac{m_{5}}{\sin I} \frac{B_{i}}{n_{i}}+\frac{3}{2}\left(\frac{1}{2} \chi_{1}-\chi_{2}\right) \sum_{\tau= \pm 1} \sum_{i \approx 0} \frac{C_{i}(\tau)}{n_{\mu}-\tau n_{i}}\right] \cos \tau \theta_{i}\right.
\end{gathered}
$$




$$
\begin{aligned}
& -\left[\frac{\left(m_{5}+2 \cos I\right)}{\sin I} \chi_{2} \sum_{\tau= \pm 1} \sum_{i \neq 0} \frac{D_{i}(\tau)}{2 n_{\mu}-\tau \theta_{i}}+\frac{1}{2}\left(\frac{1}{2} \chi_{1}-\chi_{2}\right) \sum_{\tau= \pm 1} \sum_{i \neq 0} \frac{C_{i}(\tau)}{n_{\mu}-\tau m_{i}}\right] \cos \left(2 \mu-\tau \theta_{i}\right) \\
& \left.-\frac{1}{4}\left(\phi_{1}+4 \chi_{2}-\chi_{3}\right) \sum_{\tau= \pm 1} \sum_{\neq 0} \frac{D_{i}(\tau)}{2 n_{\mu}-\tau n_{i}}\left(\cos \left(3 \mu-\tau \theta_{i}\right)+\cos \left(\mu-\tau \theta_{i}\right)\right)\right\}
\end{aligned}
$$

\section{Numerical Representation of Nutation Series}

The numerical computations of the forced nutation for angular momentum axis eqns. (47), (48), (50), (51), (52), (53), (79), (80), (82), (83), (84), (85), (86) and (87) will be carried out by using the numerical coefficient in table $1[10,17]$.

Table 1. Numerical coefficients.

\begin{tabular}{lll}
\hline Parameter & Values & Source \\
\hline$\Omega$ & $15^{\prime \prime} .0410667$ arcsec & Allen $(2000)$ \\
$\mathrm{I}$ & $84381 " .412$ & Allen $(2000)$ \\
$\mathrm{A}$ & $0.80101 \times 10^{45} \mathrm{gr.cm}^{2}$ & Allen $(2000)$ \\
$\mathrm{C}$ & $0.80365 \times 10^{45} \mathrm{grcm}^{2}$ & Allen $(2000)$ \\
$\mathrm{a}_{\mathrm{s}}$ & $0.1495979 \times 10^{45} \mathrm{~cm}$ & Allen $(2000)$ \\
$\mathrm{a}_{\mathrm{m}}$ & $0.384401 \times 10^{11} \mathrm{~cm}$ & Allen $(2000)$ \\
$k_{\circ m}^{\prime}$ & $7568^{\prime \prime} .01772 / \mathrm{Jcy}$ & Selim $(2007)$ \\
$k_{\circ S}^{\prime}$ & $3475^{\prime \prime} .18832 / \mathrm{Jcy}$ & Selim $(2007)$ \\
\hline
\end{tabular}

Table 2. Coupling between affecting forces (Nutation in Longitude and Obliquity); Unit=0.001 mas.

\begin{tabular}{lllllll}
\hline \multirow{2}{*}{ Period (days) } & \multicolumn{2}{l}{ Nutation of Andoyer plane Longitude } & \multicolumn{3}{l}{ Nutation of equatorial plane Obliquity } \\
\cline { 2 - 7 } & andoyer & oppolzer & figlon & andoyer & Oppolzer & figobli \\
\hline-6798.36 & 0.00095 & 0.00029 & 0.00123 & -0.00020 & -0.00028 & -0.00048 \\
-3399.18 & 0.00000 & 0.00002 & 0.00003 & 0.00002 & -0.00002 & -0.00000 \\
1305.47 & -0.00000 & 0.00000 & -0.00000 & 0.00000 & -0.00000 & -0.00000 \\
409.23 & 0.00000 & 0.00000 & 0.00000 & 0.00000 & -0.00000 & 0.00000 \\
365.26 & 0.00000 & -0.00000 & 0.00000 & 0.00000 & 0.00000 & 0.00000 \\
212.32 & -0.00000 & 0.00000 & -0.00000 & 0.00000 & -0.00000 & -0.00000 \\
182.62 & -0.00003 & -0.00014 & -0.00016 & -0.00014 & 0.00014 & -0.00000 \\
121.75 & -0.00000 & -0.00001 & -0.00001 & -0.00001 & 0.00001 & -0.00000 \\
117.54 & -0.00000 & 0.00000 & -0.00000 & 0.00000 & -0.00000 & -0.00000 \\
-32.61 & 0.00000 & -0.00000 & 0.00000 & -0.00000 & 0.00000 & -0.00000 \\
29.53 & -0.00000 & 0.00000 & -0.00000 & 0.00000 & -0.00000 & -0.00000 \\
-27.33 & 0.00000 & 0.00000 & 0.00000 & 0.00000 & -0.00000 & -0.00000 \\
\hline
\end{tabular}

Table 3. Affecting forces separately (Nutation in Longitude and Obliquity); Unit=0.001 mas.

\begin{tabular}{lllll}
\hline \multirow{3}{*}{ Period (days) } & \multicolumn{3}{l}{ Free Rigid } & \multicolumn{2}{l}{ Nutation of equatorial plane Obliquity } \\
\cline { 2 - 4 } & \multicolumn{2}{l}{ Nutation of Andoyer plane longitude } & andoyeroblir & figoblir \\
\cline { 2 - 4 } & andoyerlonr & 0.00000 & 0.00000 & 0.00000 \\
\hline-6798.36 & 0.00000 & 0.00000 & -0.00000 & -0.00000 \\
-3399.18 & 0.00000 & -0.00000 & -0.00000 & -0.00000 \\
1305.47 & -0.00000 & 0.00000 & -0.00000 & -0.00000 \\
409.23 & 0.00000 & 0.00000 & 0.00000 & 0.00000 \\
365.26 & 0.00000 & -0.00000 & -0.00000 & -0.00000 \\
212.32 & -0.00000 & -0.00000 & 0.00000 & 0.00000 \\
182.62 & -0.00000 & -0.00000 & 0.00000 & 0.00000 \\
121.75 & -0.00000 & -0.00000 & 0.00000 & 0.00000 \\
117.54 & -0.00000 & 0.00000 & 0.00000 & 0.00000 \\
-32.61 & 0.00000 & -0.00000 & 0.00000 & 0.00000 \\
29.53 & -0.00000 & 0.00000 & -0.00000 & -0.00000 \\
-27.33 & 0.00000 & &
\end{tabular}


Table 3. Continued.

\begin{tabular}{lllllll}
\hline \multirow{3}{*}{ Period (days) } & \multicolumn{2}{l}{ Tidal Forces } & & & & \multicolumn{3}{l}{ Nutation of equatorial plane Obliquity } \\
\cline { 2 - 7 } & \multicolumn{2}{l}{ Nutation of Andoyer plane longitude } & & andoyeroblit & opoblit & figoblit \\
\cline { 2 - 7 } & andoyerlont & oplont & figlont & 0.000000 & 0.00028 & 0.00028 \\
\hline-6798.36 & 0.00096 & 0.00000 & 0.00000 & 0.00000 & 0.00002 & 0.00002 \\
-3399.18 & 0.00000 & 0.00000 & -0.00000 & 0.00000 & 0.00000 & 0.00000 \\
1305.47 & -0.00000 & 0.00000 & 0.00000 & 0.00000 & 0.00000 & 0.00000 \\
409.23 & 0.00000 & 0.00000 & 0.00000 & 0.00000 & -0.00000 & -0.00000 \\
365.26 & 0.00000 & -0.00000 & -0.00000 & 0.000000 & 0.00000 & 0.00000 \\
212.32 & -0.00000 & 0.00000 & -0.00000 & 0.00043 & -0.00027 & 0.00016 \\
182.62 & -0.00000 & -0.00000 & -0.00000 & 0.00002 & -0.00001 & 0.00001 \\
121.75 & -0.00000 & -0.00000 & -0.00000 & 0.00000 & 0.00000 & 0.00000 \\
117.54 & -0.00000 & 0.00000 & 0.00000 & 0.00000 & -0.00000 & -0.00000 \\
-32.61 & 0.00000 & -0.00000 & -0.00000 & 0.00000 & 0.00000 & 0.00000 \\
29.53 & -0.00000 & 0.00000 & 0.00000 & 0.00000 & 0.00000 & 0.00000 \\
-27.33 & 0.00000 & 0.00000 & & & & \\
\hline
\end{tabular}

\section{Conclusion}

Numerical of nutation series for the plane perpendicular to angular momentum vector and the plane perpendicular to figure axis of Earth will be carried out for the two cases of the present theories, tidal effect's forces coupling with the geopotential and the other by neglect the coupling between affecting forces. As mention before, we taking the approximation triaxial symmetry of Earth and using the other numerical coefficient listed in table 1 (Numerical coefficients) [10, 17].

As we saw in Part 1 and 2

Tides are generated by the same forces, which cause nutation, but with the basic difference that the tidal effects depend on the elastic responses of the Earth, and in turn on the Earth's internal structure.

In part 1, tidal disturbances cause vertical deformation not exceeding 0 ". 009. The tidal distortion of the Earth's figure appears as a displacement of the plane by a few centimeters. These changes are reflected as variations in the astronomical latitude (defined as the complement of the angle between the rotation axis and the local vertical). The geocentric latitude is also affected but to a lesser extent not exceeding 0 . “ 003 .

In part 2 , we notes that tidal disturbance nearly neglected in case of free rotation of Earth, but the effected forces are that forces caused by gravitation of Moon and Sun which clear at the same periods as part 1 (periods -6798.36 and 182, 62) although the precession and nutation are less than in the case of coupling effects but they are clear.

\section{Acknowledgements}

Authors are grateful to the anonymous referee for helpful comments.

\section{References}

[1] Munck, W, H. and Macdonald, G. J. "The Rotation of the Earth". Cambridge (1960).

[2] Getino, J. and ferrandiz, J. M., A Hamiltonian theory for an elastic earth: Canonical variables and kinetic energy, Celest. Mech. 49, 303 (1990).

[3] Getino, J. and ferrandiz, J. M., A Hamiltonian theory for an elastic earth: Elastic energy of deformation, Celest. Mech. 51, 17 (1991a).

[4] Getino, J. and ferrandiz, J. M., A Hamiltonian theory for an elastic earth: First order analytical integration, Celest. Mech. 51,35 (1991b).

[5] Getino, J. and ferrandiz, J. M., A Hamiltonian theory for an elastic earth: Secular rotational acceleration, Celest. Mech. 52, 381 (1991c).

[6] Getino, J. and ferrandiz, J. M., On the effect of the mantle elasticity on the earth's rotation, Celest. Mech. 61, 117 (1995).

[7] Sauchay, J. and Kinoshita, H., Comparison of new nutation series with numerical integration, Celest. Mech. 52, 45 (1991).

[8] Ferrandiz, J. M.; Navarro, J. F., Escapa, A.; and Getino, J., Earth's Rotation: A Challenging Problem in Mathematics and Physics, Pure Appl. Geophys. 172, 57-74 (2015).

[9] Selim, H. H., Forced Nutation for The Rigid Earth Model At The First Order, NRIAG Journal of Astronomy and Geophysics, Special Issue, PP. 275-290 (2004).

[10] Selim, H. H., Hamiltonian of A Second Order Two- Layer Earth Model, Journal of The Korean Astronomical Society, 40, 49 (2007).

[11] Capitaine, N.; Mathews, P. M.; Dehant, V.; Wallace, P. T.; Lambert, S. B., On the IAU 2000/2006 precession-nutation and comparison with other models and VLBI observations, Celest Mech Dyn Astronomy, 103 (2), 179 (2009).

[12] Malkin, Z., Joint analysis of celestial pole offset and free core nutation series, J. Geodesy, Volume 91, (7), 839 (2017).

[13] Schindelegger M.; Einšpigel, D.; Salstein, D.; Böhm, J., The Global S1 Tide in Earth's Nutation, Surv Geophys, 37 (3), pp. 643 (2016).

[14] Hori, G., Theory of General Perturbation with Unspecified Canonical Variable, pub1. Astr. Soc. Japan, 18 (4), 287. (1966).

[15] Kinoshita, H., Theory of the rotation of the rigid earth, Celest. Mec, h. 15, 2. (1977).

[16] Getino, J., Forced nutations of a rigid mantle-liquid core Earth model, Geophysics J. Int., 122, 803-814, (2007).

[17] Allen's Astrophysical Quantities, fourth addition, Ed. Arthur N. Cox, Springer (2000). 